\title{
Ein weiterer Schritt in Richtung Professionalisierung
}

ETHIKKOMMISSION FÜR PHYSIOTHERAPIE Vor einem Jahr hat die Ethikkommission für physiotherapeutische Forschung des Berufsverbands ZVK ihre Arbeit aufgenommen. Ihr Vorsitzender Stefan Hegenscheidt spricht über Ziele und Aufgaben der Kommission und erklärt, wie Physiotherapeuten ihre geplante Studie ethisch prüfen lassen können.

\section{$S$}

tefan Hegenscheidt, der Vorsitzende der Ethikkommission, ist 39 Jahre alt und seit 2006 wissenschaftlicher Mitarbeiter der Physio-Akademie. Nach seiner Physiotherapieausbildung machte er eine OMT-Weiterbildung und schloss 2006 ein Masterstudium in Neuromusculoskeletal Therapy an der University of Brighton (Großbritannien) ab. Seit 2011 ist er dort Doktorand. Zudem ist er Dozent in der OMT-Weiterbildung.

Herr Hegenscheidt, bisher gab es in Deutschland keine vergleichbare Kommission für die Physiotherapie. Woran haben Sie sich bei der Gründung orientiert?

Gesetzliche Vorschriften, die bei der Gründung zu beachten sind, gibt es nicht. Wir haben daher sorgfältig recherchiert, wie sich Ethikkommissionen in der Regel zusammensetzen und wie sie arbeiten. Dabei orientierten wir uns auch an ausländischen Kommissionen, etwa den englischen, und deren $\mathrm{Er}$ fahrungen. Zudem war uns wichtig, eine große Bandbreite an Gutachtern zu haben, um möglichst viele Perspektiven einbeziehen zu können. Letztendlich sind nun Kollegen aus der Praxis, Experten für Forschungsmethoden und ethische Fragen sowie ein Patientenvertreter Mitglieder der Kommission.

Wie gehe ich vor, wenn ich mein Studienprotokoll bei Ihnen einreichen möchte?
Wir haben ein Formular entwickelt, das alle Kernaspekte umfasst, auf die wir bei der Begutachtung Wert legen. Dazu zählen beispielsweise die genaue Beschreibung der Methodik sowie Maßnahmen zum Schutz der Probanden und deren Daten. Das Formular findet man auf der Homepage der Physio-Akademie (॰ „Ethikkommission“). Wir haben übrigens die Rückmeldung bekommen, dass bereits die Arbeit mit dem Formular sehr hilfreich ist, da es zum Nachdenken über das eigene Vorgehen anregt.

\section{Und wenn ich das Formular dann ausgefüllt habe?}

Dann schicken Sie Ihren Antrag an unsere Geschäftsstelle. Sie prüft, ob Ihr Vorhaben überhaupt eine Begutachtung erfordert.

Das heißt, es gibt Projekte, bei denen eine Begutachtung durch die Kommission nicht notwendig ist?

Ja, wir haben schon Anfragen von Physiotherapeuten erhalten, die lediglich ihre Arbeit im laufenden Praxisbetrieb evaluieren wollten, zum Beispiel Messdaten aus der Trainingstherapie. Solche Anfragen begrüßen wir natürlich trotzdem. Eine ethische Begutachtung ist aber bei Daten, die ohnehin im Rahmen der Untersuchung und Therapie und nicht mit zusätzlichen Verfahren erhoben werden, nicht erforderlich.

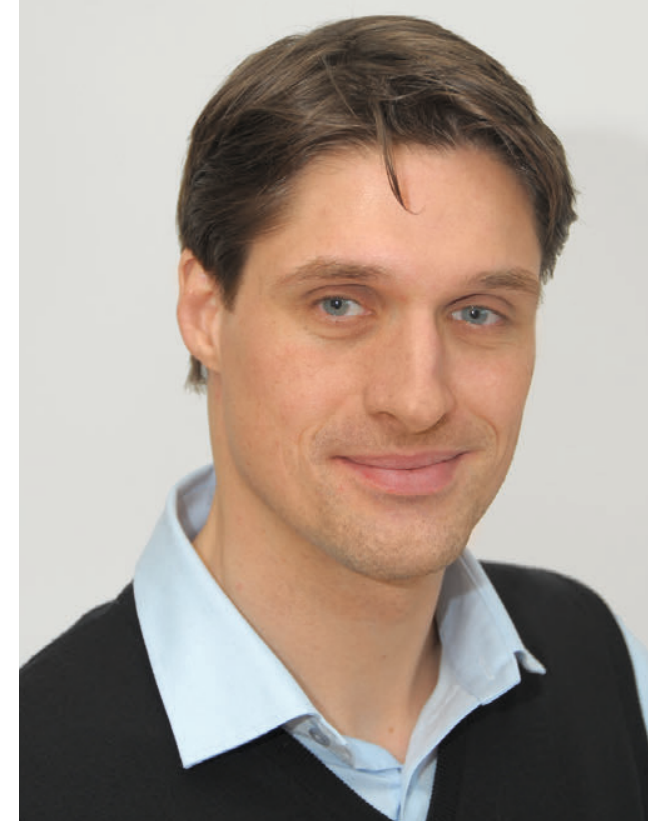

Wie läuft die Begutachtung $a b$ ?

Stellt sich heraus, dass eine Begutachtung erforderlich ist, werden von den sieben Gutachtern drei ausgelost, die das Protokoll prüfen. Dieses erhalten sie dann per E-Mail mit einer etwa dreiwöchigen Bearbeitungszeit. Den Gutachtern liegt eine Prüfungsvorlage vor, an der sie sich orientieren können. Darin trägt jeder seine Kommentare ein und schickt sie an die Geschäftsstelle zurück. Sollte ein spezieller Begutachtungsbedarf bestehen, es etwa unbedingt erforderlich sein, dass der Patientenvertreter hinzugezogen wird, wird dieser gesondert um seine Mitarbeit gebeten. Die Einzelbegutachtungen werden dann in der Geschäftsstelle zu einem gemeinsamen Statement zusammengefasst und gehen anschließend noch einmal zur Freigabe an die Gutachter. Im Anschluss erhält der Antragsteller die Rückmeldung. Der Antrag kann ohne Anmerkungen befürwortet werden oder mit kleineren bis hin zu umfassenden Überarbeitungen. Letztere erfordern eventuell auch eine erneute Vorlage, bevor die Studie beginnt. Abgelehnt haben wir bisher keinen Antrag.

Wie viele Forschungsprotokolle haben Sie bisher begutachtet?

Im letzten Jahr haben wir fünf Anträge geprüft, momentan laufen drei Begutachtungen. Bei vier weiteren war eine Prüfung nicht notwendig. 


\section{Ethikkommission - Sinn, Aufgaben und Kontakt}

Ethikkommissionen tragen dafür Sorge, dass wissenschaftliche Studien ethisch vertretbar sind. Damit schützen sie die Interessen und Rechte der Probanden und bewahren die Forschenden davor, gegen gesetzliche Regelungen zu verstoßen ( $\odot$ physiopraxis $4 / 09$, S. 22, „Ethische Unbedenklichkeit bestätigen lassen“). Eingerichtet sind sie meist bei Landesärztekammern und medizinischen Fakultäten der Hochschulen. Wollen Physiotherapeuten ein Forschungsprojekt durchführen, lehnen ärztliche Kommissionen eine Begutachtung oft ab, sofern die geplante Studie keine ärztliche Leitung hat.

Da mittlerweile jedoch immer mehr Therapeuten eigene Studien durchführen und die Physio-Akademie in Wremen zunehmend Anfragen zur ethischen Prüfung dieser Forschungsprotokolle erhielt, gründete sie im Auftrag des Deutschen Verbandes für Physio- therapie (ZVK) e.V. 2012 eine Ethikkommission speziell für Physiotherapeuten.

Sechs der sieben Kommissionsmitglieder sind Physiotherapeuten aus Wissenschaft und Praxis, ein weiteres ist Patientenvertreter. Die Gutachter arbeiten ehrenamtlich und ziehen, falls notwendig, weitere Experten (etwa Juristen) zur Beratung hinzu. Für die Begutachtung eines Studienprotokolls erhebt die Kommission eine Gebühr von 300 Euro, weitere Einnahmen hat sie nicht. Interessierte Physiotherapeuten können über ethikkommission@physio-akademie.de Kontakt aufnehmen.

Weitere Informationen zum Begutachtungsverfahren“ bietet die Homepage der Physio-Akademie: www.physio-akademie.de/forschungentwicklung/wissenschaftliche-services/ ethikkommission/.

\section{Wie lange dauert dieses Prozedere?}

Pro Antrag etwa sechs Wochen.

Geben Sie uns ein Beispiel für einen Antrag, den Sie wahrscheinlich ablehnen würden.

Nehmen wir an, man würde einer Gruppe von Patienten nach Schlaganfall eine neuartige Therapie anbieten und einer zweiten Gruppe gar keine Therapie. Ethisch bedenklich wäre, wenn man der zweiten Gruppe damit bereits bewährte, erfolgversprechende Therapien vorenthielte.

Was würde für mich als Forscher passieren, wenn Sie meinen Antrag ablehnen?

Rein rechtlich gar nichts. Bisher sind Physiotherapeuten nämlich nicht gesetzlich verpflichtet, ihre Studienprotokolle bei einer Ethikkommission einzureichen. Daher ist auch kein Physiotherapeut verpflichtet, sich an unsere Rückmeldungen zu halten. Würde er die Studie trotzdem durchführen, wäre das allerdings unethisch und auch in höchstem Maße unprofessionell. Da wir mit der Kommission ja einen Beitrag zur Professionalisierung der Physiotherapie leisten wollen, sollte physiotherapeutische Forschung unserer Meinung nach niemals ohne ethische Begutachtung erfolgen.

Sie sind der Vorsitzende der Kommission. Wie kam es zu Ihrer Mitarbeit und was reizt Sie daran?
Als Mitarbeiter der Physio-Akademie war ich wesentlich an deren Aufbau beteiligt und habe mich, als letztendlich alles stand, um die Mitgliedschaft in der Kommission beworben. Die Ethikkommission kann Gutachter vorschlagen, und der Deutsche Verband für Physiotherapie beruft diese dann. Mich reizt besonders, dass ich einen direkten Einblick in physiotherapeutische Forschungsprojekte bekomme und einen Eindruck erhalte, für welche Fragestellungen sich Kollegen interessieren beziehungsweise wie sie diese beantworten möchten.

\section{Benötigt die Kommission noch weitere perso- nelle Unterstützung?}

Momentan können wir die Anfragen mit unseren sieben Gutachtern ganz gut bewältigen. Sollte die Zahl der Anträge irgendwann steigen, möchten wir den Gutachterkreis natürlich erweitern. Wir sind offen für weitere engagierte Kollegen mit vielfältigen Expertisen. Das können zum Beispiel juristische Kenntnisse sein oder auch besondere Erfahrungen aus der Praxis, etwa mit vulnerablen Gruppen, zu denen unter anderem Menschen mit einer geistigen Behinderung oder Kinder gehören.

Das Gespräch führte Eva Trompetter. 